\title{
LIE GROUP STRUCTURES ON GROUPS OF DIFFEOMORPHISMS AND APPLICATIONS TO CR MANIFOLDS
}

\author{
M. S. BAOUENDI, L. P. ROTHSCHILD, J. WINKELMANN, D. ZAITSEV \\ Dedicated to Louis Boutet de Monvel on the occasion of his sixtieth birthday
}

\section{INTRODUCTION}

One of the main objectives of this paper is to address the following question: When is the global CR automorphism group of a CR manifold a Lie group in an appropriate topology? We give here sufficient geometric conditions on a CR manifold $M$ to guarantee that the group of all its smooth (and real-analytic when $M$ is real-analytic) CR automorphisms has the structure of a (finitedimensional) Lie group compatible with its natural topology. The results of this paper are obtained by first establishing general theorems on Lie group structures for subgroups of diffeomorphisms of a given smooth or real-analytic manifold, and then applying these results together with recent work concerning jet parametrization and complete systems for CR automorphisms. We also prove a partial converse in the real-analytic case: If the real-analytic CR automorphism group of a connected real-analytic CR manifold $M$ has the structure of a Lie group such that its isotropy group at a point $p \in M$ has finitely many connected components, then the (real-analytic) CR automorphisms satisfy so-called "finite jet determination" at $p$. That is, there is an integer $k$ such that two automorphisms of $M$ coincide if and only if their derivatives at $p$ up to order $k$ are the same.

In Section 2 we define a notion of a complete system for a set of diffeomorphisms of a manifold and show (Proposition 2.2) that it is equivalent to a notion of a jet parametrization. A closed subgroup of the diffeomorphism group of a connected manifold $M$ satisfying a complete system at every point has a Lie group structure; this is the content of Theorem 2.3. Analogous definitions and results are also obtained for subsets of germs of diffeomorphisms fixing a point (Proposition 2.8 and Theorem 2.9). The notion of complete system we use here is motivated by recent results establishing this property for CR automorphisms, due to Ebenfelt [E01] and Kim and the fourth author [KZ02] (see also Han[H97]). In the real-analytic case, results on jet parametrization were obtained by the first two authors jointly with Ebenfelt [BER97, BER99a] and by the fourth author [Z97]. The partial converse mentioned above is stated in both global and local cases in Theorems 2.4 and 2.10 .

Sections 3, 4 and 5 are devoted to the proofs of the results stated in Section 2. In Section 6 we recall basic definitions and properties of abstract and embedded CR manifolds that will guarantee the existence of a Lie group structure on the group of CR automorphisms. In particular, we recall

The first and second authors are partially supported by National Science Foundation grant DMS-01-00330. 
the definitions of finite type and finite nondegeneracy (generalizing that of Levi-nondegeneracy for a real hypersurface in a complex manifold). One motivation for considering the conditions of finite type and finite nondegeneracy is that they hold for some important examples such as the "light cone tube" $\left\{\left(\operatorname{Re} z_{1}\right)^{2}+\left(\operatorname{Re} z_{2}\right)^{2}=\left(\operatorname{Re} z_{3}\right)^{2}\right\}$, for which Levi-nondegeneracy does not hold. Another motivation is that an arbitrary smooth or real-analytic embedded generic submanifold in $\mathbb{C}^{N}$ can always be perturbed to satisfy these two conditions (see the forthcoming paper [BRZ04]). In contrast, a general real hypersurface in $\mathbb{C}^{N}$ cannot necessarily be deformed into one which is Levi-nondegenerate at every point.

Theorem 6.2 states that the global CR automorphism group of a CR manifold satisfying the conditions of finite type and finite nondegeneracy at every point has a Lie group structure. (For Levi-nondegenerate hypersurfaces, the latter result follows from the work of E. Cartan [C32], Tanaka [T67], Chern-Moser [CM74] and Kobayashi [Ko72], see also Burns-Shnider [BS77].) In Section 6 we also briefly survey results on the group of germs of local CR automorphisms fixing a point of a CR manifold. In particular, under the two nondegeneracy conditions mentioned above, such a group has a Lie group structure in the real-analytic case but may not be a Lie group in the smooth case.

\section{Complete Systems And Jet PARAmetrizATions FOR DifFEOMORPhisms}

If $M$ and $M^{\prime}$ are two smooth (resp. real-analytic) manifolds, we denote by $C^{\infty}\left(M, M^{\prime}\right)$ (resp. $\left.C^{\omega}\left(M, M^{\prime}\right)\right)$ the set of smooth (resp. real-analytic) maps from $M$ to $M^{\prime}$. We equip $C^{\infty}\left(M, M^{\prime}\right)$ (resp. $\left.C^{\omega}\left(M, M^{\prime}\right)\right)$ with its natural topology, which we shall now describe. Let $n=\operatorname{dim} M$ and $n^{\prime}=\operatorname{dim} M^{\prime}$. To simplify notation, we write $r$ for $\infty$ or $\omega$, depending on whether the manifolds $M$ and $M^{\prime}$ are smooth or real-analytic. For any local coordinate charts $(U, \varphi)$ and $(V, \psi)$ on $M$ and $M^{\prime}$ respectively, denote by

$$
C^{r}\left(M, M^{\prime} ; U, V\right):=\left\{f \in C^{r}\left(M, M^{\prime}\right): f(U) \subset V\right\},
$$

and define the map $\Theta_{U, \varphi ; V, \psi}$ by

$$
C^{r}\left(M, M^{\prime} ; U, V\right) \ni f \mapsto \Theta_{U, \varphi ; V, \psi}(f):=\psi \circ f \circ \varphi^{-1} \in C^{r}\left(\varphi(U), \mathbb{R}^{n^{\prime}}\right) .
$$

We equip $C^{r}\left(M, M^{\prime} ; U, V\right)$ with the weakest topology for which the $\Theta_{U, \varphi ; V, \psi}$ is continuous. (Here, for an open set $\Omega \subset \mathbb{R}^{n}$, the vector space $C^{\infty}\left(\Omega, \mathbb{R}^{n^{\prime}}\right)$ is equipped with the Fréchet space topology of uniform convergence of the mappings and their derivatives on compact sets. The vector space $C^{\omega}\left(\Omega, \mathbb{R}^{n^{\prime}}\right)$ is equipped with its usual topology of inductive limit of Fréchet spaces of holomorphic maps in open neighborhoods in $\mathbb{C}^{n}$ of $\Omega \subset \mathbb{R}^{n} \subset \mathbb{C}^{n}$.) We may now describe the topology of $C^{r}\left(M, M^{\prime}\right)$ as follows. A subset $\mathcal{O} \subset C^{r}\left(M, M^{\prime}\right)$ is open if and only if for all choices of local charts $(U, \varphi)$ and $(V, \psi)$ as above, $\mathcal{O} \cap C^{r}\left(M, M^{\prime} ; U, V\right)$ is open in $C^{r}\left(M, M^{\prime} ; U, V\right)$.

We equip $\operatorname{Diff}(M)$ (resp. $\left.\operatorname{Diff}^{\omega}(M)\right)$, the subset of all smooth (resp. real-analytic) maps that are diffeomorphisms from $M$ onto itself with the topology induced from $C^{\infty}(M, M)$ (resp. $\left.C^{\omega}(M, M)\right)$. Note that if a sequence $\left(f_{j}\right)$ converges to $f$ in $\operatorname{Diff}(M)\left(\operatorname{resp}\right.$. Diff $\left.{ }^{\omega}(M)\right)$ then the sequence $\left(f_{j}^{-1}\right)$ converges to $f^{-1}$ in the same topology. (See e.g. Lemma 3.2 below.) It can be further shown that $\operatorname{Diff}(M)$ (resp. $\left.\operatorname{Diff}^{\omega}(M)\right)$ is a topological group. 
For a nonnegative integer $k$, we use the notation $J^{k}\left(M, M^{\prime}\right)$ for the space of all $k$-jets of smooth maps from $M$ to $M^{\prime}$ and write for simplicity $J^{k}(M):=J^{k}(M, M)$ (see e.g. [GG73] for basic definitions and properties). Furthermore, we denote by $G^{k}(M) \subset J^{k}(M)$ the open subset of all invertible jets. If $p, p^{\prime} \in M$, we denote by $J_{p}^{k}(M) \subset J^{k}(M)$ and $G_{p}^{k}(M) \subset G^{k}(M)$ the subsets of jets with source $p$, and by $G_{p, p^{\prime}}^{k}(M) \subset G_{p}^{k}(M)$ the subset of jets with source $p$ and target $p^{\prime}$. Note that $G_{p, p}^{k}(M)$ is a submanifold of $G^{k}(M)$ equipped with a Lie group structure whose multiplication is given by composition. For a jet $\Lambda \in J^{k}\left(M, M^{\prime}\right)$, denote by $\Lambda^{(s)}$ its projection to $J^{s}\left(M, M^{\prime}\right)$ for any $0 \leq s \leq k$. Given any smooth map $f: M \rightarrow M^{\prime}$ and $x \in M$, we denote by $j_{x}^{k} f \in J^{k}\left(M, M^{\prime}\right)$ its $k$-jet at $x .^{1}$

2.1. Results for global diffeomorphisms. Our main tools for establishing Lie group structures on subgroups of $\operatorname{Diff}(M)$ and $\operatorname{Diff}^{\omega}(M)$ are the so-called complete systems and closely related notions of jet parametrizations.

Definition 2.1. Let $M$ be a smooth (resp. real-analytic) manifold. A subset $\mathcal{S} \subset \operatorname{Diff}(M)$ (resp. $\left.\mathcal{S} \subset \operatorname{Diff}^{\omega}(M)\right)$ is said to satisfy a smooth (resp. real-analytic) complete system at a point $x_{0} \in M$ if there exists a nonnegative integer $k$ such that, for every $\Lambda_{0} \in G_{x_{0}}^{k}(M)$, there exists a neighborhood $\Omega$ of $\Lambda_{0}$ in $G^{k}(M)$ and a smooth (resp. real-analytic) map $\Phi: \Omega \rightarrow J^{k+1}(M)$ such that

$$
j_{x}^{k+1} f=\Phi\left(j_{x}^{k} f\right),
$$

for any $x \in M$ and $f \in \mathcal{S}$ with $j_{x}^{k} f \in \Omega$. In this case we shall say that the order of the complete system $(2.1)$ is $k+1$. We say that $\mathcal{S}$ satisfies a complete system if it satisfies a complete system at every point in $M$.

The reader should be warned that, for a given $f \in \mathcal{S}$ in the above definition, the open subset of $x \in M$ for which $j_{x}^{k} f \in \Omega$, may be empty or, even if not empty, may not contain the point $x_{0}$.

The following proposition gives a necessary and sufficient condition for $\mathcal{S}$ to satisfy a complete system of order $k+1$ at $x_{0}$ in terms of a local $k$-jet parametrization of mappings in $\mathcal{S}$.

Proposition 2.2. In the setting of Definition 2.1 the subset $\mathcal{S}$ satisfies a smooth (resp. realanalytic) complete system of order $k+1$ at $x_{0} \in M$ if and only if, for every $\Lambda_{0} \in G_{x_{0}}^{k}(M)$, there exist neighborhoods $\Omega^{\prime}$ of $x_{0}$ in $M, \Omega^{\prime \prime}$ of $\Lambda_{0}$ in $G^{k}(M)$, and a smooth (resp. real-analytic) map $\Psi: \Omega^{\prime} \times \Omega^{\prime \prime} \rightarrow M$ such that

$$
f(x)=\Psi\left(x, j_{y}^{k} f\right)
$$

for any $x \in \Omega^{\prime}$, any $y \in M$ and any $f \in \mathcal{S}$ with $j_{y}^{k} f \in \Omega^{\prime \prime}$.

As usual we shall say that a Lie group $G$ acts smoothly (resp. real-analytically) on a smooth (resp. real-analytic) manifold $M$ if the action map $G \times M \rightarrow M$ is smooth (resp. real-analytic). One of the main results of this section is the following.

\footnotetext{
${ }^{1}$ If $M$ is an open subset of $\mathbb{R}^{n}$, and $M^{\prime}$ an open subset of $\mathbb{R}^{n^{\prime}}$, then $J^{k}\left(M, M^{\prime}\right) \cong M \times M^{\prime} \times \mathbb{R}^{N}$, where $N$ is $n^{\prime}$ times the number of multiindices $\alpha \in \mathbb{N}^{n}$, with $0<|\alpha| \leq k$, and $j_{x}^{k} f=\left(x, f(x),\left(\partial^{\alpha} f(x)\right)_{0<|\alpha| \leq k}\right)$.
} 
Theorem 2.3. Let $M$ be a smooth (resp. real-analytic) connected manifold and $G \subset \operatorname{Diff}(M)$ (resp. $G \subset \operatorname{Diff}^{\omega}(M)$ ) be a closed subgroup satisfying a smooth (resp. real-analytic) complete system. Then $G$, equipped with the induced topology of $\operatorname{Diff}(M)$ (resp. $\operatorname{Diff}^{\omega}(M)$ ), has a (unique) structure as a Lie group acting smoothly (resp. real-analytically) on $M$.

Note that if a topological group has a Lie group structure, the latter is necessarily unique (see e.g. [V74]). The proofs of Proposition 2.2 and Theorem 2.3 will be given in Section 3. We also have the following partial converse of Theorem 2.3.

Theorem 2.4. Let $M$ be a connected real-analytic manifold and $G \subset \operatorname{Diff}^{\omega}(M)$ be a subgroup equipped with a Lie group structure acting real-analytically on $M$. Fix a point $p \in M$ and assume that the Lie subgroup $G_{p}:=\{g \in G: g(p)=p\}$ has finitely many connected components. Then there exists an integer $k$ such that for any two elements $g_{1}, g_{2} \in G$,

$$
g_{1}=g_{2} \text { if and only if } j_{p}^{k} g_{1}=j_{p}^{k} g_{2} .
$$

Observe that the assumption that $G_{p}$ has finitely many components is automatically satisfied when $M$ is a real-algebraic manifold and $G$ is a real-algebraic group acting algebraically on $M$.

The proof of Theorem 2.4 will be given in Section 5. We would like to point out that the conclusion of Theorem 2.4 does not hold without the assumption that $G_{p}$ has finitely many components, as can be seen by the following example.

Example 2.5. Let $M:=\mathbb{R}^{2}, p=0$,

$$
G:=\left\{g: \mathbb{R}^{2} \rightarrow \mathbb{R}^{2}: g(x, y)=(x, y+P(x)), P(x) \in \mathbb{Z}[x]\right\},
$$

where $\mathbb{Z}[x]$ denotes the ring of polynomials in one variable with integer coefficients. It is easy to see that the topology of $\operatorname{Diff}^{\omega}(M)$ induces the discrete topology on $G \subset \operatorname{Diff}^{\omega}(M)$. Hence $G$, as well as $G_{p}$, are (zero-dimensional) Lie groups with infinitely many components, and the conclusion of Theorem 2.4 obviously does not hold.

We should mention that Theorem 2.4 does not hold in the smooth category, i.e. with $M$ a smooth manifold and $G \subset \operatorname{Diff}(M)$, as can be easily seen by the following modification of Example 2.5. Example 2.6. Let $M:=\mathbb{R}^{2}, p=0$,

$$
G:=\left\{g: \mathbb{R}^{2} \rightarrow \mathbb{R}^{2}: g(x, y)=(x, y+c \chi(x)), c \in \mathbb{R}\right\},
$$

where $\chi(x)$ is a smooth nonzero real function on $\mathbb{R}$ vanishing of infinite order at 0 . It is easy to see that $G \subset \operatorname{Diff}(M)$ is a closed subgroup and a Lie group (isomorphic to $\mathbb{R}$ ) in the induced topology. However the conclusion of Theorem 2.4 does not hold.

In the context of Theorem 2.4 one can ask whether the stronger conclusion that $G$ satisfies a complete system at $p$ also holds. The authors know of no example where finite jet determination (in the sense of Theorem 2.4) holds without the existence of a complete system.

2.2. Results for germs of diffeomorphisms. We consider now local versions of Theorems 2.3 and 2.4. Let $M$ be again a smooth (resp. real-analytic) manifold and fix a point $p \in M$. We denote by $\operatorname{Diff}(M, p)$ (resp. $\left.\operatorname{Diff}^{\omega}(M, p)\right)$ the germs at $p$ of all local smooth (resp. real-analytic) diffeomorphisms of $M$ fixing $p$. Similarly denote by $C^{\infty}(M, p)\left(\right.$ resp. $\left.C^{\omega}(M, p)\right)$ the germs at $p$ of 
all smooth (resp. real-analytic) maps fixing $p$. We equip the latter sets with the following topology. A subset $\mathcal{O} \subset C^{\infty}(M, p)$ (resp. $\mathcal{O} \subset C^{\omega}(M, p)$ ) is said to be open if and only if, for every open neighborhood $U$ of $p$ in $M$, the subset $\left\{f \in C^{\infty}(U, M):[f] \in \mathcal{O}\right\}\left(\operatorname{resp} .\left\{f \in C^{\omega}(U, M):[f] \in \mathcal{O}\right\}\right)$ is open in $C^{\infty}(U, M)$ (resp. $\left.C^{\omega}(U, M)\right)$, where $[f]$ denotes the germ of $f$ at $p$. We further equip $\operatorname{Diff}(M, p) \subset C^{\infty}(M, p)$ (resp. Diff $\left.{ }^{\omega}(M, p) \subset C^{\omega}(M, p)\right)$ with the induced topology. In contrast to $\operatorname{Diff}^{\omega}(M, p)$, the topology on $\operatorname{Diff}(M, p)$ is not Hausdorff and therefore will not be considered. Note that a sequence $\left(f_{n}\right)$ in $\operatorname{Diff}^{\omega}(M, p)$ converges to $f$ if $f$ and $f_{n}$ have representatives (denoted by the same letters) defined in a fixed open neighborhood $U$ of $p$ in $M$ and such that $f_{n} \rightarrow f$ in $C^{\omega}(U, M)$.

The following is a local version of Definition 2.1.

Definition 2.7. Let $M$ be a smooth (resp. real-analytic) manifold and $p \in M$. A subset $\mathcal{S} \subset$ $\operatorname{Diff}(M, p)$ (resp. $\mathcal{S} \subset \operatorname{Diff}^{\omega}(M, p)$ ) is said to satisfy a smooth (resp. real-analytic) local complete system if there exists a nonnegative integer $k$ such that, for every $\Lambda_{0} \in G_{p}^{k}(M)$, there exists a neighborhood $\Omega$ of $\Lambda_{0}$ in $G^{k}(M)$ and a smooth (resp. real-analytic) map $\Phi: \Omega \rightarrow J^{k+1}(M)$ such that

$$
j_{x}^{k+1} f=\Phi\left(j_{x}^{k} f\right),
$$

for any $f$ representing a germ in $\mathcal{S}$ with $j_{p}^{k} f \in \Omega$ and $x \in M$ sufficiently close to $p$.

Note that if $f$ is a germ at $p \in M$ of a smooth map from $M$ into itself, then $j^{k} f: x \mapsto j_{x}^{k} f$ is a germ at $p$ of a section from $M$ to $J^{k}(M)$. If $\Phi: \Omega \rightarrow J^{k+1}(M)$ is as in Definition 2.7 with $j_{p}^{k} f \in \Omega$, then $\Phi \circ\left(j^{k} f\right)$ is a germ at $p$ of a smooth map from $M$ to $J^{k+1}(M)$. Equality (2.3) simply means the equality of the two germs, $j^{k+1} f$ and $\Phi \circ\left(j^{k} f\right)$, at $p$.

As in the global case in Proposition 2.2, we have the following characterization of local complete systems in terms of local jet parametrizations.

Proposition 2.8. In the setting of Definition 2.7 the subset $\mathcal{S} \subset \operatorname{Diff}(M, p)\left(r e s p . \operatorname{Diff}^{\omega}(M)\right)$ satisfies a smooth (resp. real-analytic) local complete system if and only if there exists an integer $k$ such that, for every $\Lambda_{0} \in G_{p}^{k}(M)$, there exist neighborhoods $\Omega^{\prime}$ of $p$ in $M, \Omega^{\prime \prime}$ of $\Lambda_{0}$ in $G_{p}^{k}(M)$, and a smooth (resp. real-analytic) map $\Psi: \Omega^{\prime} \times \Omega^{\prime \prime} \rightarrow M$ such that

$$
f(\cdot)=\Psi\left(\cdot, j_{p}^{k} f\right)
$$

for any $f \in \mathcal{S}$ with $j_{p}^{k} f \in \Omega^{\prime \prime}$, where the equality in (2.4) holds in the sense of germs at $p$.

For a subgroup $G \subset \operatorname{Diff}^{\omega}(M, p)$ equipped with a Lie group structure, we shall say that $G$ acts real-analytically on $M$ if, for any $g_{0} \in G$, there is an open neighborhood $U^{\prime} \times U^{\prime \prime}$ of $\left(p, g_{0}\right)$ in $M \times G$ and a real-analytic map $\theta: U^{\prime} \times U^{\prime \prime} \rightarrow M$ such that for any $g \in U^{\prime \prime}$, the germ at $p$ of the map $x \mapsto \theta(x, g)$ coincides with $g$.

We now state the following local version of Theorem 2.3.

Theorem 2.9. Let $M$ be a real-analytic manifold and $p \in M$. Let $G \subset \operatorname{Diff}^{\omega}(M, p)$ be a closed subgroup satisfying a smooth (resp. real-analytic) local complete system. Then $G$, equipped with the induced topology of $\operatorname{Diff}^{\omega}(M, p)$, has a (unique) structure as a Lie group acting real-analytically on $M$. 
The proofs of Proposition 2.8 and Theorem 2.9 will be given in Section 4. As in the global case we also have the following local analogue of Theorem 2.4.

Theorem 2.10. Suppose that $M$ is a real-analytic manifold, and let $p$ be a point in $M$. Let $G \subset \operatorname{Diff}^{\omega}(M, p)$ be a subgroup equipped with a Lie group structure acting real-analytically on $M$. Assume that $G$ has finitely many connected components. Then there exists an integer $k$ such that for any two elements $g_{1}, g_{2} \in G$,

$$
g_{1}=g_{2} \text { if and only if } j_{p}^{k} g_{1}=j_{p}^{k} g_{2} .
$$

The proof of Theorem 2.10 will be given in Section 5 .

\section{LiE GROUP STRUCTURES FOR GLOBAL DIFFEOMORPHISMS}

In this section we shall give proofs of the results about subsets of (global) diffeomorphisms stated in Section 2.

Proof of Proposition 2.2. We start by assuming that (2.2) holds in the setting of the proposition. By differentiating (2.2) we obtain the complete system (2.1) with

$$
\Omega:=\left\{j_{x}^{k} h \in G^{k}(M): x \in \Omega^{\prime}, j_{x}^{k} h \in \Omega^{\prime \prime}\right\}, \quad \Phi\left(j_{x}^{k} h\right):=j_{x}^{k+1} \Psi\left(\cdot, j_{x}^{k} h\right)
$$

proving one implication of the proposition.

To prove the converse, we now assume that (2.1) holds. Let $x_{0}^{\prime} \in M$ be the target of the given jet $\Lambda_{0}$. We take local coordinates $\left(x_{1}, \ldots, x_{n}\right)$ (resp. $\left.\left(x_{1}^{\prime}, \ldots, x_{n}^{\prime}\right)\right)$ on $M$ vanishing at $x_{0}$ (resp. at $\left.x_{0}^{\prime}\right)$. By shrinking $\Omega$ if necessary, we may assume that $\Omega \subset G^{k}(M)$ is a coordinate neighborhood of $\Lambda_{0}$ with respect to the induced coordinates on $J^{k}(M)$. Hence an element $\Lambda \in \Omega$ has coordinates $\left(x, \Lambda^{\alpha}\right)_{0 \leq|\alpha| \leq k}$ with $x, \Lambda^{\alpha} \in \mathbb{R}^{n}$. Here $\alpha$ is a multiindex in $\mathbb{Z}_{+}^{n}$. It follows from (2.1) that we have, in particular,

$$
\partial^{\alpha} f(x)=\Phi_{\alpha}\left(x,\left(\partial^{\beta} f(x)\right)_{0 \leq|\beta| \leq k}\right), \quad|\alpha|=k+1,
$$

where $\Phi_{\alpha}$ is a smooth (resp. real-analytic) function defined in $\Omega$. By setting $f_{\beta}(x):=\partial^{\beta} f(x)$, we obtain from (3.1) a system of the form

$$
\partial_{x_{j}} f_{\beta}(x)=r_{j \beta}\left(x,\left(f_{\gamma}(x)\right)_{0 \leq|\gamma| \leq k}\right), \quad 1 \leq j \leq n, 0 \leq|\beta| \leq k .
$$

For $l=1, \ldots, n$, denote by $\chi^{l}(y, t, \Lambda)$ the unique solution of the initial value problem for the ordinary differential equation

$$
\frac{d g}{d t}(t)=r_{l}\left(y_{1}, \ldots, y_{l-1}, t, y_{l+1}, \ldots, y_{n}, g(t)\right), \quad g\left(y_{l}\right)=\Lambda,
$$

where $g(t)=\left(g_{\beta}(t)\right)_{0 \leq|\beta| \leq k}$ with each $g_{\beta}(t)$ being an $\mathbb{R}^{n}$-valued function of one variable. Similarly $r_{l}=\left(r_{l \beta}\right)$ with $r_{j \beta}$ given by (3.2) and $\Lambda=\left(\Lambda^{\beta}\right)$ with $\Lambda^{\beta} \in \mathbb{R}^{n}$. It follows from the standard theory of ordinary differential equations with parameters that the function $\chi^{l}$ is uniquely determined for $(y, t, \Lambda)=\left(y_{1}, \ldots, y_{n}, t, \Lambda\right)$ near $\left(0,0, \Lambda_{0}\right)$ and is smooth (resp. real-analytic). Using the notation $u_{s}:=\left(x_{1}, \ldots, x_{s-1}, y_{s}, \ldots, y_{n}\right), s=1, \ldots, n$, (and hence $\left.u_{1}=y\right)$, we define

$$
\chi(x, y, \Lambda):=\chi^{n}\left(u_{n}, x_{n}, \ldots \chi^{2}\left(u_{2}, x_{2}, \chi^{1}\left(u_{1}, x_{1}, \Lambda\right)\right) \ldots\right) .
$$


Hence $\chi(x, y, \Lambda)=\left(\chi_{\beta}(x, y, \Lambda)\right)_{0 \leq|\beta| \leq k}$ is again defined for $(x, y, \Lambda)$ sufficiently close to $\left(0,0, \Lambda_{0}\right)$. We claim that the component $\Psi(x, y, \Lambda):=\chi_{0}(x, y, \Lambda)$ satisfies the desired conclusion of the proposition. Indeed, if $f \in \mathcal{S}$ and $\left(x, j_{y}^{k} f\right)$ is sufficiently close to $\left(x_{0}, \Lambda_{0}\right)$ (independently of the choice of $f)$, then (2.1) and hence (3.2) is satisfied. Thus $g(t):=\left(f_{\beta}\left(y_{1}, \ldots, y_{l-1}, t, y_{l+1}, \ldots, y_{n}\right)\right)$ solves the initial value problem (3.3) with $\Lambda=\left(f_{\beta}(y)\right)$. Hence $\left(f_{\beta}(x)\right)=\chi\left(x, y,\left(f_{\gamma}(y)\right)\right.$ by the construction of $\chi$. The claim follows by taking $\beta=0$. The proof of the proposition is complete.

We shall now give the proof of Theorem 2.3 in the smooth case. We will not give the details of the proof of the real-analytic case, since it is completely analogous. We start with the following proposition, which will be needed for the proof of the theorem.

Proposition 3.1. Let the assumptions of Theorem 2.3 be satisfied. Then, for any $p \in M$, there exists an integer $k \geq 1$ such that the mapping

$$
j_{p}^{k}: G \rightarrow G_{p}^{k}(M), \quad g \mapsto j_{p}^{k} g,
$$

is a homeomorphism onto a closed subset of $G_{p}^{k}(M)$.

The following known consequence of the implicit function theorem will be used in the proof of Proposition 3.1.

Lemma 3.2. Let $U \subset \mathbb{R}^{N}$ be an open set and $f_{n}: U \rightarrow \mathbb{R}^{N}$ a sequence of smooth (resp. realanalytic) maps which converges to a smooth (resp. real-analytic) map $f$ in the $C^{\infty}\left(U, \mathbb{R}^{N}\right)$ (resp. $\left.C^{\omega}\left(U, \mathbb{R}^{N}\right)\right)$ topology. Let $p \in U$ and assume that the Jacobian $\operatorname{Jac} f(p) \neq 0$. Then there exists an open neighborhood $V \subset \mathbb{R}^{N}$ of $f(p)$ such that $f^{-1}$ and $f_{n}^{-1}$ are defined in $V$ for $n$ sufficiently large. Moreover the sequence $\left(f_{n}^{-1}\right)$ converges to $f^{-1}$ in the topology of $C^{\infty}\left(V, \mathbb{R}^{N}\right)$ (resp. $\left.C^{\omega}\left(V, \mathbb{R}^{N}\right)\right)$.

The main step in the proof of Proposition 3.1 is given by the following lemma.

Lemma 3.3. Let $G$ satisfy the assumptions of Theorem 2.3. Fix $p \in M$ and assume that $G$ satisfies a complete system of order $k+1$ at $p$ with $k \geq 1$. If $\left(f_{n}\right)$ is a sequence in $G$ with $\lim _{n \rightarrow \infty} j_{p}^{k} f_{n}=\lambda \in G_{p}^{k}(M)$, then $f_{n}$ converges to an element $f$ in $G$ (and hence $j_{p}^{k} f=\lambda$ ).

Proof. We use Proposition 2.2 with $x_{0}=p$ and $\Lambda_{0}=\lambda$. Let $\Omega^{\prime}, \Omega^{\prime \prime}$ and $\Psi$ be given by the proposition. For $n$ large enough we have $j_{p}^{k} f_{n} \in \Omega^{\prime \prime}$. Hence for such $n$ we have

$$
f_{n}(x)=\Psi\left(x, j_{p}^{k} f_{n}\right), \quad \text { for all } x \in \Omega^{\prime} .
$$

When $n \rightarrow \infty$ the right hand side of $(3.5)$ converges to $f^{[p]}(x):=\Psi(x, \lambda)$ in $C^{\infty}\left(\Omega^{\prime}, M\right)$. We conclude from (3.5) that the sequence $\left(f_{n}\right)$ converges to $f^{[p]}$ in $C^{\infty}\left(\Omega^{\prime}, M\right)$. Since this implies, in particular, that $\lim _{n \rightarrow \infty} j_{p}^{k} f_{n}=j_{p}^{k} f^{[p]}=\lambda$, we conclude that the $\operatorname{Jacobian} \operatorname{Jac} f^{[p]}(p) \neq 0$.

Denote by $\mathcal{O}$ the set of points $q \in M$ such that the sequence $\left(f_{n}\right)$ converges in the $C^{\infty}$ topology in a neighborhood of $q$ and such that $\operatorname{Jac}\left(\lim f_{n}\right)(q) \neq 0$. It is clear that $\mathcal{O}$ is open and contains $p$ by the above argument. We must show that $\mathcal{O}$ is closed. For this, let $q_{0}$ be in the closure of $\mathcal{O}$ in $M$. We shall apply Proposition 2.2 with $x_{0}=q_{0}, k_{0}$ being the corresponding integer (i.e. $\mathcal{S}$ satisfies a complete system of order $k_{0}+1$ at $\left.x_{0}\right)$, and $\Lambda_{0}=j_{x_{0}}^{k_{0}}$ id $\in G_{x_{0}}^{k_{0}}(M)$, where id is the identity mapping on $M$. We let again $\Omega^{\prime}, \Omega^{\prime \prime}$ and $\Psi$ be given by Proposition 2.2 for the latter choice of $x_{0}$ and $\Lambda_{0}$. Fix $q \in \mathcal{O}$ such that $j_{q}^{k_{0}}$ id $\in \Omega^{\prime \prime}$. Set $f^{[q]}(x):=\lim _{n \rightarrow \infty} f_{n}(x)$ which is defined 
in a neighborhood of $q$ by the definition of $\mathcal{O}$. By Lemma 3.2, it follows that there exists $n_{0}$ such that for $n, m \geq n_{0}$, the following holds:

$$
j_{q}^{k_{0}}\left(f_{n}^{-1} \circ f_{m}\right) \in \Omega^{\prime \prime}, \quad j_{q}^{k_{0}}\left(f_{n}^{-1} \circ f^{[q]}\right) \in \Omega^{\prime \prime} .
$$

Therefore we have by Proposition 2.2,

$$
f_{n_{0}}^{-1}\left(f_{m}(x)\right)=\Psi\left(x, j_{q}^{k_{0}}\left(f_{n_{0}}^{-1} \circ f_{m}\right)\right), \quad \text { for all } x \in \Omega^{\prime} \text { and } m \geq n_{0} .
$$

Since the right-hand side converges in $C^{\infty}\left(\Omega^{\prime}, M\right)$ as $m \rightarrow \infty$, we conclude that the sequence $\left(f_{m}\right)$ also converges in $C^{\infty}\left(\Omega^{\prime}, M\right)$ to a map $f^{\left[q_{0}\right]}$.

Recall that $q_{0} \in \Omega^{\prime}$. Hence, to show that $q_{0} \in \mathcal{O}$, it remains to prove that $\operatorname{Jac} f^{\left[q_{0}\right]}\left(q_{0}\right) \neq 0$. For this, we again make use of Proposition 2.2 with $x_{0}=q_{0}^{\prime}:=f^{\left[q_{0}\right]}\left(q_{0}\right)$, and $\Lambda_{0}=j_{x_{0}}^{k_{1}}$ id, assuming that $\mathcal{S}$ satisfies a complete system of order $k_{1}+1$ at $x_{0}$. We denote again by $\Omega^{\prime}, \Omega^{\prime \prime}$ and $\Psi$ the data associated to this choice given by the proposition. Let $q_{1} \in \mathcal{O}$ be sufficiently close to $q_{0}$ such that $j_{q^{\prime}}^{k_{1}}$ id $\in \Omega^{\prime \prime}$ holds with $q^{\prime}:=f^{\left[q_{0}\right]}\left(q_{1}\right)$. If $f^{\left[q_{1}\right]}$ denotes the limit of the sequence $\left(f_{n}\right)$ defined in a neighborhood of $q_{1}$, by Lemma 3.2 , there exist $n_{1}$ such that, for $n, m \geq n_{1}$, we have

$$
j_{q^{\prime}}^{k_{1}}\left(f_{n} \circ f_{m}^{-1}\right) \in \Omega^{\prime \prime}, \quad j_{q^{\prime}}^{k_{1}}\left(f_{n_{1}} \circ\left(f^{\left[q_{1}\right]}\right)^{-1}\right) \in \Omega^{\prime \prime} .
$$

Note that $f^{\left[q_{1}\right]}(x)=f^{\left[q_{0}\right]}(x)$ for all $x$ in a neighborhood of $q_{1}$. By Proposition 2.2, we have for $m \geq n_{1}$,

$$
f_{n_{1}}\left(f_{m}^{-1}(x)\right)=\Psi\left(x, j_{q^{\prime}}^{k_{1}}\left(f_{n_{1}} \circ f_{m}^{-1}\right)\right), \quad \text { for all } x \in \Omega^{\prime} .
$$

By the choice of $q_{1}$ and $q^{\prime}$, again the right-hand side converges in a neighborhood of $q_{0}^{\prime}$ and hence $\left(f_{m}^{-1}(x)\right)$ also converges in the same neighborhood of $q_{0}^{\prime}$. Denote by $g$ its limit. Since $g\left(f^{\left[q_{0}\right]}(x)\right)=x$ for $x$ in a neighborhood of $q_{0}$, we conclude that $\operatorname{Jac} f^{\left[q_{0}\right]}\left(q_{0}\right) \neq 0$. Hence $q_{0} \in \mathcal{O}$ proving that $\mathcal{O}=M$ by connectedness of $M$. Thus the sequence $\left(f_{n}\right)$ converges in $C^{\infty}(M, M)$ to a mapping $f$ with nowhere vanishing Jacobian.

By using Lemma 3.2 again, we see that, for $n$ sufficiently large, the sequence $\left(f_{n}^{-1}\right)$ converges in a fixed neighborhood of $p^{\prime}:=f(p)$ in the $C^{\infty}$ topology. Since, for any $l$, the jets $j_{p^{\prime}}^{l} f_{n}^{-1}$ converge in $G^{l}(M)$, we may apply the above argument to the sequence $\left(f_{n}^{-1}\right)$ instead of $\left(f_{n}\right)$ to conclude that $\left(f_{n}^{-1}\right)$ converges to a map $g: M \rightarrow M$ in the $C^{\infty}$ topology. Then it follows that $g \circ f=f \circ g=$ id and therefore $f \in \operatorname{Diff}(M)$. Since $G$ is assumed to be closed in $\operatorname{Diff}(M)$, we conclude that $f \in G$ completing the proof of the lemma.

Proof of Proposition 3.1. By the assumption of the proposition, given a point $p \in M, G$ satisfies a complete system of order $k+1$ at $p$ for some integer $k$ (see Definition 2.1 with $x_{0}=p$ ). We shall show that the map (3.4), with this value of $k$, satisfies the conclusion of Proposition 3.1.

We first show that the map (3.4) is injective. For this, let $g_{1}, g_{2} \in G$ with $j_{p}^{k} g_{1}=j_{p}^{k} g_{2}$. By Proposition 2.2, we conclude that $g_{1}$ and $g_{2}$ agree in a neighborhood of $p$ in $M$. Then the set

$$
V:=\left\{q \in M: g_{1}(x)=g_{2}(x) \text { for } x \text { in a neighborhood of } q\right\}
$$

contains $p$ and is open again by Proposition 2.2. By the connectedness of $M$, it suffices to show that $V$ is closed in $M$. For this, let $q_{0}$ be in the closure of $V$. Then, by Proposition 2.2 with $x_{0}=q_{0}$, 
there exists an integer $k_{0}$ and, for $\Lambda_{0}:=j_{q_{0}}^{k_{0}} g_{1}$, a map $\Psi: \Omega^{\prime} \times \Omega^{\prime \prime} \rightarrow M$, for which (2.2) holds. Then, for $y \in V, j_{y}^{k_{0}} g_{1}=j_{y}^{k_{0}} g_{2}$ and hence, for $y \in V$ sufficiently close to $q_{0}, g_{i}(x)=\Psi\left(x, j_{y}^{k_{0}} g_{i}\right)$ holds for $x \in \Omega^{\prime}$ and $i=1,2$. In particular, we obtain $g_{1}(x)=g_{2}(x)$ for $x$ in a neighborhood of $q_{0}$. This proves that $q_{0} \in V$ and hence the map (3.4) is injective. As an immediate consequence of Lemma 3.3, we see that the image $j_{p}^{k}(G)$ is closed in $G_{p}^{k}(M)$. The map $j_{p}^{k}$ in $(3.4)$ is clearly continuous. Since we have shown that it is injective, the rest of the proof of the proposition is a consequence of Lemma 3.3.

As a direct corollary of Lemma 3.3, we obtain the following.

Corollary 3.4. Under the assumptions of Theorem 2.3, let $k_{0}$ be the minimum of the integers $k$ given by Definition 2.1 as the point $x_{0} \in M$ varies. Then the topologies induced on $G$ by $C^{\infty}(M, M)$ and $C^{k_{0}}(M, M)$ coincide.

We complete the proof of Theorem 2.3 by applying the following theorem of BochnerMontgomery [BM46].

Theorem 3.5 (Bochner-Montgomery). Let $G$ be a locally compact topological group acting continuously on a smooth manifold $M$ by smooth diffeomorphisms. Suppose that the identity is the only element of $G$ which fixes every point of a nonempty open subset of $M$. Then $G$ is a Lie group and the action $G \times M \rightarrow M$ is smooth.

Proof of Theorem 2.3. We apply Theorem 3.5 to our situation. Since, for every $p \in M$ and every integer $k$, the invertible jet space fiber $G_{p}^{k}(M)$ is a manifold, it follows from Proposition 3.1 that $G$ is locally compact. Moreover, since the map (3.4) is injective, the second assumption of Theorem 3.5 also holds. The proof is completed by applying Theorem 3.5.

\section{LiE GROUP STRUCTURES FOR GERMS OF DIFFEOMORPHISMS}

In this section we shall give proofs of the local results stated in Section 2.

Proof of Proposition 2.8. The proof of the fact that (2.3) implies (2.4) is analogous to the corresponding part in the proof of Proposition 2.2. We shall prove that (2.4) implies (2.3). Since the statements are local, we may assume that $M=\mathbb{R}^{n}$ and $p=0$. As in the proof of Proposition 2.2, we take local coordinates $x=\left(x_{1}, \ldots, x_{n}\right)$ on $\mathbb{R}^{n}$ inducing coordinates $(x, \Lambda)=\left(x,\left(\Lambda_{\beta}\right)_{0 \leq|\beta| \leq k}\right)$ on the jet space $J^{k}\left(\mathbb{R}^{n}\right) \cong \mathbb{R}^{n} \times \mathbb{R}^{N}$, where $N$ is the number of the multiindices $\beta$ with $0 \leq|\beta| \leq k$. Let $\Omega^{\prime}, \Omega^{\prime \prime}$ and $\Psi$ be as in Proposition 2.8. Then we have $0 \in \Omega^{\prime} \subset \mathbb{R}^{n}$ and $\Lambda_{0} \in \Omega^{\prime \prime} \subset \mathbb{R}^{N}$. We begin by changing $\Psi$ to obtain $\widetilde{\Psi}$ with the property

$$
\partial_{x}^{\alpha} \widetilde{\Psi}(0, \Lambda)=\Lambda_{\alpha}, \quad \Lambda \in \Omega^{\prime \prime}
$$

For this, we take

$$
\widetilde{\Psi}(x, \Lambda):=\Psi(x, \Lambda)+\sum_{0 \leq|\beta| \leq k}\left(\Lambda_{\beta}-\partial_{x}^{\beta} \Psi(0, \Lambda)\right) \frac{x^{\beta}}{\beta !} .
$$

Then (4.1) holds and (2.4) still holds with $\Psi$ replaced by $\widetilde{\Psi}$. 
Consider the map

$$
\Omega^{\prime} \times \Omega^{\prime \prime} \ni(x, \Lambda) \mapsto \Theta(x, \Lambda):=\left(x,\left(\partial_{x}^{\alpha} \widetilde{\Psi}(x, \Lambda)\right)_{0 \leq|\alpha| \leq k}\right) \in \mathbb{R}^{n} \times \mathbb{R}^{N} .
$$

By (4.1), $\Theta(0, \Lambda) \equiv(0, \Lambda)$. By the implicit function theorem, there exists a smooth (resp. real-analytic) function $\chi(x, \Lambda)$ defined in a neighborhood of $\left(0, \Lambda_{0}\right)$ in $\mathbb{R}^{n} \times \mathbb{R}^{N}$ satisfying $\Theta(x, \chi(x, \Lambda)) \equiv \Lambda$. Then

$$
\Phi(x, \Lambda):=\left(x,\left(\left.\partial_{y}^{\alpha} \widetilde{\Psi}(y, \chi(x, \Lambda))\right|_{y=x}\right)_{0 \leq|\alpha| \leq k+1}\right)
$$

satisfies the desired conclusion.

Proof of Theorem 2.9. We want to show that the image of the continuous group homomorphism

$$
G \ni g \mapsto j_{p}^{k} g \in G_{p, p}^{k}(M)
$$

is closed. Since this image is a subgroup of $G_{p, p}^{k}(M)$, it suffices to show that it is closed in a neighborhood of the identity. For this, we apply Proposition 2.8 to $\mathcal{S}:=G$ and $\Lambda_{0}:=j_{p}^{k}$ id. We claim that the image of $G$ is closed in $\Omega^{\prime \prime} \subset G_{p}^{k}(M)$. Indeed, given a sequence $g_{n} \in G$ with $j_{p}^{k} g_{n} \rightarrow \lambda \in \Omega^{\prime \prime}$ as $n \rightarrow \infty$, the sequence $\tilde{g}_{n}(x):=\Psi\left(x, j_{p}^{k} g_{n}\right)$ consists of representatives of $g_{n}$ defined in $\Omega^{\prime}$, a uniform neighborhood of $p$. Clearly $\tilde{g}_{n}(x)$ converges to $\tilde{g}(x):=\Psi(x, \lambda)$ in $C^{\infty}\left(\Omega^{\prime}, M\right)$ (resp. $\left.C^{\omega}\left(\Omega^{\prime}, M\right)\right)$. It follows that the germ $g_{n}$ converges in $\operatorname{Diff}(M, p)$ (resp. $\left.\operatorname{Diff}^{\omega}(M, p)\right)$ to $g$, the germ of $\tilde{g}$ at $p$, and hence $\lambda=j_{p}^{k} g$. Since $G$ is closed $\operatorname{in} \operatorname{Diff}(M, p)$ (resp. $\operatorname{Diff}^{\omega}(M, p)$ ), it follows that $g \in G$ proving the claim. The same argument also shows that the homomorphism (4.3) is a homeomorphism onto its image. Since a closed subgroup of a Lie group is a Lie subgroup, it follows that $G$ has a (necessarily unique) Lie group structure (see e.g. [V74]). Furthermore, the conclusion of Proposition 2.8 also implies that the action of $G$ is smooth (resp. real-analytic). The proof of the theorem is complete.

\section{Finite Jet Determination For Lie GRoup ACTIONS}

The purpose of this section is to prove the following finite jet determination result which is slightly more general than Theorem 2.10 and from which Theorem 2.4 will follow.

Proposition 5.1. Let $M$ be a connected real-analytic manifold and $p$ a point in $M$. Suppose that $G$ is a Lie group with finitely many connected components equipped with a continuous injective homomorphism $\iota: G \rightarrow \operatorname{Diff}^{\omega}(M, p)$. Then there exists a number $k$ such that, for $g_{1}, g_{2} \in G$, $j_{p}^{k}\left(\iota\left(g_{1}\right)\right)=j_{p}^{k}\left(\iota\left(g_{2}\right)\right)$ if and only if $g_{1}=g_{2}$.

As a first step, we need some basic facts about jet groups. For every positive integer $k$, consider the subgroup

$$
L_{k}:=\left\{f \in \operatorname{Diff}^{\omega}(M, p): j_{p}^{k} f=j_{p}^{k} \text { id }\right\} .
$$

If $\left(x_{1}, \ldots, x_{n}\right)$ are local coordinates on $M$ vanishing at $p$, then every $\varphi \in L_{k}$ can be written as

$$
\varphi:\left(x_{1}, \ldots, x_{n}\right) \mapsto\left(x_{1}+f_{1}(x), \ldots, x_{n}+f_{n}(x)\right)
$$


where $x=\left(x_{1}, \ldots, x_{n}\right)$ and $f_{i}(x)=o\left(|x|^{k}\right), x \rightarrow 0$. As a consequence we obtain that $L^{k} / L^{k+1}$ is a commutative group which can be identified with the additive group of all $\mathbb{R}^{n}$-valued homogeneous polynomials on $\mathbb{R}^{n}$ of degree $k+1$. In particular $L^{k} / L^{k+1}$ is isomorphic to the additive group of a finite-dimensional real vector space. Thus we have established:

Lemma 5.2. Let $(M, p)$ be a germ of a real-analytic manifold and $L_{k}$ be the subgroup given by (5.1). Then $L^{k} / L^{k+1}$ is a torsion-free commutative group for all $k \geq 1$.

We shall also need the following well-known fact from Lie group theory for which we give a proof for the reader's convenience:

Lemma 5.3. The fundamental group of a connected Lie group is commutative and finitely generated.

Proof. Let $G$ be a connected Lie group and $\pi: \widetilde{G} \rightarrow G$ the universal covering group of $G$. The kernel $H$ of $\pi$ is therefore a normal discrete subgroup of $\widetilde{G}$ that is isomorphic to the fundamental group of $G$. Hence $g h g^{-1} \in H$ for all $g \in \widetilde{G}$ and $h \in H$. Since $\widetilde{G}$ is connected and $H$ is discrete, it follows that $g h g^{-1}=h$ for all $g$ and $h$ as above proving the commutativity of $H$ and hence of the fundamental group of $G$.

To show that the fundamental group of $G$ is finitely generated, consider a maximal compact subgroup $K$ of $G$. Then $G$ is homeomorphic to $K \times \mathbb{R}^{l}$ for some nonnegative integer $l$, in particular, $G$ and $K$ have isomorphic fundamental groups. Since $K$ is a compact manifold, its fundamental group is necessarily finitely generated. This completes the proof of the lemma.

Now we are ready to prove Proposition 5.1.

Proof of Proposition 5.1. For $k \in \mathbb{N}$, let

$$
H_{k}:=\left\{g \in G: j_{p}^{k}(\iota(g))=j_{p}^{k} \mathrm{id}\right\} .
$$

Since $H_{k}$ is the kernel of the homomorphism $G \ni g \mapsto j_{p}^{k}(\iota(g)) \in G_{p}^{k}(M),\left(H_{k}\right)$ is a descending sequence of normal closed Lie subgroups of $G$. Moreover, since $\iota$ is injective and $\iota(g)$ is real-analytic for any $g \in G$, it follows that

$$
\bigcap_{k} H_{k}=\{e\}
$$

where $e$ is the unit in $G$. In fact, we shall show that there exists a number $k \in \mathbb{N}$ such that $H_{k}=\{e\}$.

Note that $\operatorname{dim} H_{k}$ is a decreasing sequence of nonnegative integers and set $d_{0}:=\lim _{k \rightarrow \infty} \operatorname{dim} H_{k}$. Then there is a number $n_{0} \in \mathbb{N}$ such that $\operatorname{dim} H_{k}=d_{0}$ for all $k \geq n_{0}$. Since $H_{k} \supset H_{k+1}$ for all $k$, this implies that the connected component $H_{k}^{0}$ of $e$ in $H_{k}$ is the same for all $k \geq n_{0}$. Combined with (5.3) this implies that $H_{k}^{0}=\{e\}$ and hence that $H_{k}$ is discrete for $k \geq n_{0}$.

From now on, consider only $k$ with $k \geq n_{0}$. Denote by $G^{0}$ the connected component of $e$ in $G$ and set $I_{k}:=H_{k} \cap G^{0}$. Since $H_{k}$ is discrete and normal in $G$, we conclude that $I_{k}$ is a discrete normal subgroup of the connected Lie group $G^{0}$. Thus $G^{0} / I_{k}$ is a connected Lie group and the canonical map $G^{0} \rightarrow G^{0} / I_{k}$ is a covering map. By lifting each loop through the unit in $G^{0} / I_{k}$ 
to a (unique) path in $G^{0}$ starting from $e$, we obtain a surjective group homomorphism from the fundamental group $\pi_{1}\left(G^{0} / I_{k}\right)$ to $I_{k}$. Since, by Lemma 5.3, $\pi_{1}\left(G^{0} / I_{k}\right)$ is necessarily a finitely generated commutative group, it follows that $I_{k}$ is also finitely generated and commutative.

Recall that the (free) rank of a finitely generated commutative group $A$, regarded as a $\mathbb{Z}$-module, is the maximal number of $\mathbb{Z}$-linearly independent elements in $A$. Recall also that, by the main structure theorem for finitely generated commutative groups (see e.g. [DF91]), $A$ is isomorphic to a direct sum of cyclic groups $\mathbb{Z}^{r} \oplus \mathbb{Z}_{a_{1}} \oplus \cdots \oplus \mathbb{Z}_{a_{l}}$, where $\mathbb{Z}_{a}$ is the cyclic group of order $a$. In this case $\operatorname{rank}(A)=r$. Since $\left(I_{k}\right)$ is a decreasing sequence of finitely generated commutative groups, there exists a natural number $n_{1} \geq n_{0}$ such that $\operatorname{rank}\left(I_{k}\right)=\operatorname{rank}\left(I_{k+1}\right)$ for all $k \geq n_{1}$. Then it follows from the definition of the rank that $I_{k} / I_{k+1}$ consists of elements of finite order. From now on, consider only $k$ with $k \geq n_{1}$. By construction, there exists an embedding $I_{k} / I_{k+1} \hookrightarrow L_{k} / L_{k+1}$. Since $L_{k} / L_{k+1}$ does not contain any element of finite order, it follows that $I_{k} / I_{k+1}=0$. Combined with (5.3) this implies that $I_{k}=\{e\}$.

Recall that $I_{k}=G^{0} \cap H_{k}$. Thus $I_{k}=\{e\}$ implies that the natural projection $G \rightarrow G / G_{0}$ restricts to an injective group homomorphism from $H_{k}$ to $G / G^{0}$. Since $G$ has finitely many components by assumption, it follows that $G / G^{0}$ is a finite group and hence $H_{k}$ is also finite for $k \geq n_{1}$. Thus the $\left(H_{k}\right)$ form a descending sequence of finite groups. In view of $(5.3)$, it follows that $H_{k}=\{e\}$ for sufficiently large $k \in \mathbb{N}$.

To complete the proof of Proposition 5.1, choose $k$ with $H_{k}=\{e\}$ and suppose that, for $g_{1}, g_{2} \in G, j_{p}^{k}\left(\iota\left(g_{1}\right)\right)=j_{p}^{k}\left(\iota\left(g_{2}\right)\right)$ holds. Then for $g:=g_{1}^{-1} \circ g_{2}$, we have $j_{p}^{k}(\iota(g))=j_{p}^{k}$ id. Hence $g \in H_{k}=\{e\}$ which implies $g_{1}=g_{2}$ as desired.

Proof of Theorem 2.4. We apply Proposition 5.1 to the group $G_{p}$ and the obvious homomorphism $\iota: G_{p} \rightarrow \operatorname{Diff}^{\omega}(M, p)$. Let $k$ be given by the conclusion of the proposition. Then, if for $g_{1}, g_{2} \in G$, we have $j_{p}^{k} g_{1}=j_{p}^{k} g_{2}$, then $g:=g_{1}^{-1} \circ g_{2} \in G_{p}$ satisfies $j_{p}^{k} g=j_{p}^{k}$ id. By the conclusion of Proposition 5.1, we conclude that $g=$ id proving $g_{1}=g_{2}$ as desired.

\section{Applichtions to CR automorphism groups of CR manifolds}

In this section we shall apply the results of Section 2 to the case of CR automorphism groups of CR manifolds. Recall (see e.g. [Bo91, BER99b] for more details) that an (abstract) CR manifold $M$ is a smooth manifold equipped with a complex vector subbundle $\mathcal{V}$ of the complexified tangent bundle $\mathbb{C} T M$ of $M$ such that $\mathcal{V} \cap \overline{\mathcal{V}}=0$ and $[\mathcal{V}, \mathcal{V}] \subset \mathcal{V}$. The subbundle $\mathcal{V} \subset \mathbb{C} T M$ is then called the $C R$ bundle of $M$. The (complex) fiber dimension of $\mathcal{V}$ is called the $C R$ dimension of $M$ and the (fiber) codimension of $\mathcal{V} \oplus \overline{\mathcal{V}}$ in $\mathbb{C} T M$ the $C R$ codimension of $M$. In case both $M$ and $\mathcal{V}$ are real-analytic, $M$ is called a real-analytic CR manifold.

An important class of CR manifolds is that of embedded ones. If $X$ is a complex manifold and $M \subset X$ a smooth real submanifold such that

$$
\mathcal{V}:=\left(\left.T^{0,1} X\right|_{M}\right) \cap \mathbb{C} T M
$$

is a subbundle of $\mathbb{C} T M$, where $T^{0,1} X$ is the bundle of $(0,1)$ tangent vectors on $X$, then $M$ equipped with subbundle $\mathcal{V}$ defined above is a CR manifold. It is known (see [AF79]) that a real-analytic 
CR manifold can be always considered as embedded in a complex manifold $X$ with

$$
\operatorname{dim}_{\mathbb{C}} X=\mathrm{CR} \operatorname{dim} M+\mathrm{CR} \operatorname{codim} M .
$$

In case $M$ is embedded in a complex manifold $X$ and (6.1) holds, then $M$ is called a generic submanifold of $X$. (A smooth abstract CR manifold may not be even locally embeddable in any $\mathbb{C}^{N}$, see e.g. [Bo91]).

If $M$ and $M^{\prime}$ are two smooth $\mathrm{CR}$ manifolds with CR bundles $\mathcal{V}$ and $\mathcal{V}^{\prime}$ respectively, a $C R$ map $f: M \rightarrow M^{\prime}$ is a smooth map satisfying $f_{*} \mathcal{V} \subset \mathcal{V}^{\prime}$, where $f_{*}: \mathbb{C} T M \rightarrow \mathbb{C} T M^{\prime}$ is the induced pushforward map. A $C R$ automorphism of $M$ is a $\mathrm{CR}$ map from $M$ to itself which is also a diffeomorphism. Observe that in this case the inverse map is also CR. We denote by $\operatorname{Aut}_{\mathrm{CR}}(M) \subset$ $\operatorname{Diff}(M)$ the subgroup of all smooth CR automorphisms of $M$ equipped with the induced topology. When $M$ is a real-analytic CR manifold, we also denote by $\operatorname{Aut}_{\mathrm{CR}}^{\omega}(M) \subset \operatorname{Diff}^{\omega}(M)$ the subgroup of all real-analytic CR automorphisms of $M$.

In general, $\operatorname{Aut}_{\mathrm{CR}}(M)$ (or Aut ${ }_{\mathrm{CR}}^{\omega}(M)$ in case $M$ is real-analytic) may not be a (finite-dimensional) Lie group. Indeed, for the embedded CR manifold $M:=\mathbb{R}^{n} \subset \mathbb{C}^{n}$ (and hence $\mathcal{V}=0$ ) we have $\operatorname{Aut}_{\mathrm{CR}}(M)=\operatorname{Diff}(M)$ and $\operatorname{Aut}_{\mathrm{CR}}^{\omega}(M)=\operatorname{Diff}^{\omega}(M)$. On the other hand, it is known that, if $M$ is a Levi-nondegenerate smooth hypersurface in $\mathbb{C}^{N}, N>1$, then $\operatorname{Aut}_{\mathrm{CR}}(M)$ (and also Aut ${ }_{\mathrm{CR}}^{\omega}(M)$ ) is a Lie group (see [C32, T67, CM74, BS77]).

It is clear from the above that some nondegeneracy conditions have to be imposed on a CR manifold in order for $\operatorname{Aut}_{\mathrm{CR}}(M)$ and $\mathrm{Aut}_{\mathrm{CR}}^{\omega}(M)$ to have Lie group structures (compatible with their topologies). One of the conditions we impose here, generalizing Levi-nondegeneracy, is that of finite nondegeneracy, whose definition we recall. Let $M \subset \mathbb{C}^{N}$ be a smooth generic submanifold and $p \in M$. If $d$ is the CR codimension of $M$, then $M$ is a real submanifold of codimension $d$ in $\mathbb{C}^{N}$. Let $\rho=\left(\rho^{1}, \ldots, \rho^{d}\right)$ be a smooth defining function for $M$ in a neighborhood of $p$ with linearly independent complex differentials $\partial \rho^{1}, \ldots, \partial \rho^{d}$. Then $M$ is said to be finitely nondegenerate if

$$
\operatorname{span}_{\mathbb{C}}\left\{L_{1} \ldots L_{k} \rho_{Z}^{j}(p): k \geq 0,1 \leq j \leq d\right\}=\mathbb{C}^{N}
$$

where the span is taken over all collections of smooth $(0,1)$ vector fields $L_{1}, \ldots, L_{k}$ on $M$ defined in a neighborhood of $p$. Here each $\rho_{Z}^{j}$ is regarded as the vector in $\mathbb{C}^{N}$ whose components are $\partial \rho^{j} / \partial Z_{r}$, $1 \leq r \leq N$, where $Z=\left(Z_{1}, \ldots, Z_{N}\right)$ are coordinates in $\mathbb{C}^{N}$. We further call $M$ l-nondegenerate at $p$ or say that $M$ is finitely nondegenerate of order $l$ if $l$ is the smallest integer for which (6.2) still holds under the additional restriction $k \leq l$. In particular, a hypersurface $M \subset \mathbb{C}^{N}$ is Levinondegenerate at $p$ if and only if it is 1 -nondegenerate at $p$. The reader can check that the above definitions are independent of the choice of local holomorphic coordinates $Z$ in $\mathbb{C}^{N}$ and of the defining function $\rho$. For the notion of finite nondegeneracy for abstract $\mathrm{CR}$ manifolds we refer to [BER99b]. Another way of defining this notion for an abstract CR manifold $M$ is to reduce to the embedded case by means of "approximate embeddings" in $\mathbb{C}^{N}$ for $N:=\mathrm{CR} \operatorname{dim} M+\mathrm{CR} \operatorname{codim} M$ (see [KZ02] for this approach).

We recall also the notion of finite type in the sense of Kohn and Bloom-Graham. A CR manifold $M$ is of finite type at a point $p$ if the Lie algebra generated by the $(1,0)$ and the $(0,1)$ smooth vector fields on $M$ spans the complex tangent space of $M$ at $p$. For hypersurfaces $M \subset \mathbb{C}^{N}, N>1$, 
(and, more generally, for abstract CR manifolds of CR codimension one and CR dimension at least one), it can be seen that finite nondegeneracy at $p$ implies finite type at $p$.

For CR manifolds with the above nondegeneracy conditions the following result is known.

Theorem 6.1. Let $M$ be a smooth (resp. real-analytic) CR manifold of CR codimension d, which is l-nondegenerate and of finite type at a point $p \in M$. Then the subgroup $\operatorname{Aut}_{\mathrm{CR}}(M) \subset \operatorname{Diff}(M)$ (resp. Aut ${ }_{\mathrm{CR}}^{\omega}(M) \subset \operatorname{Diff}^{\omega}(M)$ ) satisfies a smooth (resp. real-analytic) complete system at $p$ of order $k+1$ with $k:=2(d+1) l$.

Theorem 6.1 as stated above was proved by S.-Y. Kim and the fourth author [KZ02]. The embedded hypersurface case was previously established by Ebenfelt [E01]. Previous results on complete systems for CR mappings between real-analytic Levi-nondegenerate hypersurfaces were obtained in [H97]. For $M$ real-analytic, Theorem 6.1 is essentially contained in the work of the first two authors jointly with Ebenfelt [BER99a] (see also [BER97, Z97]).

6.1. Global CR automorphisms. Using Theorem 6.1 we obtain the following application of Theorem 2.3 to the global CR automorphism group of a CR manifold.

Theorem 6.2. Let $M$ be a smooth (resp. real-analytic) CR manifold, which is finitely nondegenerate and of finite type at every point. Assume that $M$ has finitely many connected components. Then $\operatorname{Aut}_{\mathrm{CR}}(M)$ (resp. Aut ${ }_{\mathrm{CR}}^{\omega}(M)$ ), equipped with the induced topology of $\operatorname{Diff}(M)$ (resp. Diff $\left.{ }^{\omega}(M)\right)$, has a (unique) structure of a Lie group acting smoothly (resp. real-analytically) on $M$.

In the case where $M$ is a compact real-analytic CR manifold satisfying the assumptions of Theorem 6.2, this result was proved in [Z97]. We note that in Theorem 6.2 no upper bound, as $p$ varies in $M$, is imposed on either the order of finite nondegeneracy at $p$, nor on the minimal length of commutators of $(1,0)$ and $(0,1)$ vector fields needed in the definition of finite type at $p$. In fact, both of these numbers can be unbounded.

Proof of Theorem 6.2. We give the proof only for $\operatorname{Aut}_{\mathrm{CR}}(M)$ since the case of $\mathrm{Aut}_{\mathrm{CR}}^{\omega}(M)$ is completely analogous. If $M$ is connected, Theorem 6.2 is an immediate consequence of Theorems 6.1 and 2.3. When $M$ has finitely many connected components, $M_{1}, \ldots, M_{l}$, it follows from the above that each $\operatorname{Aut}_{\mathrm{CR}}\left(M_{i}\right), i=1, \ldots, l$, is a Lie group. For each $g \in \operatorname{Aut}_{\mathrm{CR}}(M)$, there is a permutation $\sigma_{g}$ of $\{1, \ldots, l\}$ such that $g\left(M_{i}\right)=M_{\sigma_{g}(i)}, i=1, \ldots, l$. Then for any permutation $\sigma$ of $\{1, \ldots, l\}$, the set $\left\{g \in \operatorname{Aut}_{\mathrm{CR}}(M): \sigma_{g}=\sigma\right\}$ is a coset of the Lie group $\operatorname{Aut}_{\mathrm{CR}}\left(M_{1}\right) \times \cdots \times \operatorname{Aut}_{\mathrm{CR}}\left(M_{l}\right)$. Hence Aut $_{\mathrm{CR}}(M)$ is a Lie group as a finite (disjoint) union of cosets of a Lie group.

The assumption of finiteness of the number of components in Theorem 6.2 cannot be removed. Indeed, if $M$ has infinitely many components $M_{\alpha}, \alpha \in A$, such that each $\operatorname{Aut}_{\mathrm{CR}}\left(M_{\alpha}\right)$ is a Lie group of positive dimension, then group $\operatorname{Aut}_{\mathrm{CR}}(M)$ contains as a subgroup the infinite product $\prod_{\alpha \in A}$ Aut $_{\mathrm{CR}}\left(M_{\alpha}\right)$ and hence is not a (finite-dimensional) Lie group.

If $M$ is real-analytic and satisfies the assumptions of Theorem 6.2 then it follows from results of [BJT85], using also [Tu88], that the groups $\operatorname{Aut}_{\mathrm{CR}}(M)$ and $\operatorname{Aut}_{\mathrm{CR}}^{\omega}(M)$ coincide. However, in general, for a real-analytic CR manifold, these groups may be different even when both groups have Lie group structures compatible with their topologies, as is shown by the following example. 
Example 6.3. Let $Q \subset \mathbb{C}^{2}$ be the quadric given by $\operatorname{Im} w=|z|^{2}$ and

$$
\widehat{Q}:=\{(z, w) \in Q: z \notin S\}, \quad S:=[-1,1] \cup[0, i] \subset \mathbb{C} .
$$

All (smooth) CR automorphisms between open subsets of $Q$ are known (see e.g. [CM74]) to be restrictions to $Q$ of birational maps of $\mathbb{C}^{2}$ of the form $\Phi \circ \Psi$ with

$$
\begin{gathered}
\Phi(z, w)=\left(z+z_{0}, w+w_{0}+2 i \operatorname{Re}\left(z \bar{z}_{0}\right)\right), \quad\left(z_{0}, w_{0}\right) \in Q, \\
\Psi(z, w)=\frac{\left(\lambda(z+a w),|\lambda|^{2} w\right)}{1-2 i z \bar{a}-\left(r+i|a|^{2}\right) w}, \quad \lambda \in \mathbb{C} \backslash\{0\}, a \in \mathbb{C}, r \in \mathbb{R} .
\end{gathered}
$$

(Observe that every $\Psi$ of the form (6.4) is defined on all of $Q$ except at most one point.) Hence we can identify the CR automorphisms of $\widehat{Q}$ with their birational extensions to $Q$. Then $\operatorname{Aut}_{\mathrm{CR}}(\widehat{Q})=$ $\left\{f \in \operatorname{Aut}_{\mathrm{CR}}(Q): f(\widehat{S})=\widehat{S}\right\}$ with $\widehat{S}:=\{(z, w) \in Q: z \in S\}$, and it is then straightforward to check that

$$
\operatorname{Aut}_{\mathrm{CR}}(\widehat{Q})=\operatorname{Aut}_{\mathrm{CR}}^{\omega}(\widehat{Q})=\left\{\varphi_{u}: u \in \mathbb{R}\right\}, \quad \text { where } \varphi_{u}(z, w):=(z, w+u) .
$$

Let $\chi \in C^{\infty}(\mathbb{R})$ with $\chi(t)=0$ for $t \leq 3 / 2$ and $\chi(t)>0$ for $t>3 / 2$. Define a CR submanifold $M \subset \mathbb{C}^{3}$ by

$$
M:=((1,2) \times \widehat{Q}) \backslash F,
$$

where $F$ is the closure in $\mathbb{C}^{3}$ of the subset $\left\{\left(t, t, n \chi(t)+i t^{2}\right): t \in(1,2), n \in \mathbb{Z}\right\}$. Given any $f=$ $\left(f_{1}, f_{2}\right) \in \operatorname{Aut}_{\mathrm{CR}}(M)$, it follows from the definition that $f_{1}: M \rightarrow \mathbb{R}$ is a CR map and hence (since $\widehat{Q}$ is of finite type) $f_{1}=f_{1}(t)$ for $t \in(1,2)$, i.e. $f_{1}$ depends only on the first argument. For $t \in(1,2)$, the map $(z, w) \mapsto f_{2}(t, z, w)$ extends to a map in $\operatorname{Aut}_{\mathrm{CR}}(\widehat{Q})$ and hence, by (6.5), $f_{2}(t, z, w)=(z, w+u(t))$ for some smooth function $u(t)$. Thus $f=\left(f_{1}, t_{2}\right)$ extends to a $\mathrm{CR}$ automorphism $(t, z, w) \mapsto\left(f_{1}(t), z, w+u(t)\right)$ of $(1,2) \times \widehat{Q}$ that preserves $F$. From here it is straightforward to check that

$$
\operatorname{Aut}_{\mathrm{CR}}(M)=\left\{\Theta_{n}: n \in \mathbb{Z}\right\}, \quad \Theta_{n}(t, z, w):=(t, z, w+n \chi(t)) .
$$

Hence $\operatorname{Aut}_{\mathrm{CR}}^{\omega}(M)$ consists only of the identity map. Both $\operatorname{Aut}_{\mathrm{CR}}(M)$ and $\operatorname{Aut}_{\mathrm{CR}}^{\omega}(M)$ are (zerodimensional) Lie groups. Note that $M$ is finitely nondegenerate at every point but not of finite type at any point.

Example 6.3 furthermore shows that $\operatorname{Aut}_{\mathrm{CR}}(M)$ may have a (compatible) Lie group structure without satisfying a complete system. Indeed, at any point of the form $p_{0}=\left(3 / 2, z_{0}, w_{0}\right) \in M$, one has $j_{p_{0}}^{k} \Theta_{n}=j_{p_{0}}^{k}$ id for all $k$ and $n$ and hence even finite jet determination does not hold for Aut $_{\mathrm{CR}}(M)$. On the other hand, $\operatorname{Aut}_{\mathrm{CR}}^{\omega}(M)=\{\mathrm{id}\}$ clearly satisfies an (analytic) complete system in the sense of Definition 2.1.

It may also happen for a real-analytic CR manifold $M$ that $\operatorname{Aut}_{\mathrm{CR}}^{\omega}(M)$ is a Lie group while $\operatorname{Aut}_{\mathrm{CR}}(M)$ is not, as is shown by the following modification of Example 6.3. 
Example 6.4. We take

$$
M:=((1,2) \times \widehat{Q}) \backslash \widehat{F},
$$

where $\widehat{F}$ is the closure in $\mathbb{C}^{3}$ of the subset $\left\{\left(t, t, n \chi(t)+i t^{2}\right): 3 / 2 \leq t<2, n \in \mathbb{Z}\right\}$, where $\widehat{Q}$ and $\chi(t)$ are as in Example 6.3. Then the same arguments as in Example 6.3 show that $\operatorname{Aut}_{\mathrm{CR}}^{\omega}(M)=$ $\{$ id $\}$. However, for any function $\alpha \in C^{\infty}(\mathbb{R})$ with support in $(1,3 / 2)$, the mapping $(t, z, w) \mapsto$ $(t, z, w+\alpha(t))$ is in $\operatorname{Aut}_{\mathrm{CR}}(M)$. Hence $\operatorname{Aut}_{\mathrm{CR}}(M)$ is not a (finite-dimensional) Lie group.

An immediate application of Theorem 2.4 gives the following.

Corollary 6.5. Let $M$ be a connected real-analytic $C R$ manifold with $p \in M$, and assume that the subgroup of $\operatorname{Aut}_{\mathrm{CR}}^{\omega}(M)$ fixing $p$ has finitely many connected components. Then there exists an integer $k$ such that for any two elements $g_{1}, g_{2} \in \operatorname{Aut}_{\mathrm{CR}}^{\omega}(M)$,

$$
g_{1}=g_{2} \text { if and only if } j_{p}^{k} g_{1}=j_{p}^{k} g_{2} \text {. }
$$

The authors do not know if, under the assumptions of Corollary 6.5, the stronger conclusion that $\operatorname{Aut}_{\mathrm{CR}}^{\omega}(M)$ satisfies a complete system at $p$ also holds.

6.2. Germs of local CR automorphisms. If $M$ is a smooth (resp. real-analytic ) CR manifold and $p \in M$, we denote by $\operatorname{Aut}_{\mathrm{CR}}(M, p) \subset \operatorname{Diff}(M, p)\left(\operatorname{resp} . \operatorname{Aut}_{\mathrm{CR}}^{\omega}(M, p) \subset \operatorname{Diff}^{\omega}(M, p)\right)$ the group of all germs at $p$ of local smooth (rep. real-analytic) CR automorphisms of $M$ fixing $p$. We consider on $\operatorname{Aut}_{\mathrm{CR}}^{\omega}(M, p)$ the topology induced by $\operatorname{Diff}^{\omega}(M, p)$. Since the general results for groups of germs of local diffeomorphisms given in Section 2.2 are only for the real-analytic case, we shall first discuss this case and survey recent results.

Theorem 6.6. Let $M$ be a real-analytic CR manifold of CR codimension $d$ which is $\ell$ nondegenerate and of finite type at a point $p \in M$. Then with $k:=(d+1) \ell$ the mapping

$$
\operatorname{Aut}_{\mathrm{CR}}^{\omega}(M, p) \ni f \mapsto j_{p}^{k} f \in G_{p, p}^{k}(M)
$$

is an injective homeomorphic group homomorphism onto a totally real real-algebraic Lie subgroup of $G_{p, p}^{k}(M)$. Hence Aut $_{\mathrm{CR}}^{\omega}(M, p)$ has the structure of a real-algebraic Lie group.

Theorem 6.6, as stated above, was proved by the first two authors jointly with Ebenfelt in [BER99a]. An earlier result by the fourth author in [Z97] proved that the mapping (6.6) with $k=$ $2(d+1) \ell$ is an injective homeomorphic group homomorphism onto a closed Lie subgroup of $G_{p, p}^{k}(M)$ (The real hypersurface case was treated in [BER97]). The main tool for proving Theorem 6.6 is a local jet parametrization in the sense of Proposition 2.8.

For a real-analytic hypersurface $M \subset \mathbb{C}^{2}$, it has recently been proved in [ELZ03] that $\operatorname{Aut}_{\mathrm{CR}}^{\omega}(M, p)$ has a Lie group structure under the single assumption that $M$ is of finite type at $p$ (that is, without the condition that $M$ is finitely nondegenerate at $p$ ). More partial results are known in the direction of Theorem 6.6 stating only the injectivity of the map (6.6) for suitable $k$ [BER00, BMR02, ELZ03, Kw01].

In contrast to the real-analytic case, the statement analogous to that of Theorem 6.6 does not hold for general smooth $\mathrm{CR}$ manifolds even under the strongest possible conditions (e.g. if $M$ is a strongly pseudoconvex hypersurface in $\mathbb{C}^{2}$ ). In fact, it is shown in [KZ03] that there exists a 
smooth strongly pseudoconvex hypersurface $M \subset \mathbb{C}^{2}$ and a point $p \in M$ such that the image of the map

$$
\operatorname{Aut}_{\mathrm{CR}}(M, p) \ni f \mapsto j_{p}^{k} f \in G_{p, p}^{k}(M)
$$

is not closed for any integer $k$ (observe that the map (6.7) is known to be injective for $k \geq 2$ (see $[\mathrm{C} 32, \mathrm{CM} 74]))$. This is also in contrast with the global automorphism group $\operatorname{Aut}_{\mathrm{CR}}(M)$, where the mapping

$$
\operatorname{Aut}_{\mathrm{CR}}(M) \ni f \mapsto j_{p}^{k} f \in G_{p}^{k}(M)
$$

has always closed image for suitable $k$ under the assumptions of Theorem 6.2 on $M$ (see Proposi-

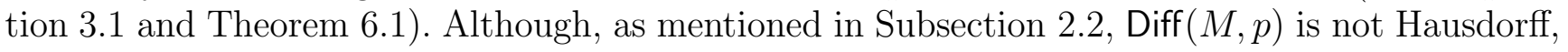
it is easy to see that its subset $\operatorname{Aut}_{\mathrm{CR}}(M, p)$ is Hausdorff in the induced topology whenever the (continuous) map (6.7) is injective for some $k$. If, moreover, $\operatorname{Aut}_{\mathrm{CR}}(M, p)$ satisfies a smooth local complete system of order $k+1$ in the sense of Definition 2.7, then it follows from Proposition 2.8 that the induced topology from $\operatorname{Diff}(M, p)$ coincides with the one induced by the injection (6.7). Hence the mentioned examples from [KZ03] show that $\operatorname{Aut}_{\mathrm{CR}}(M, p)$ may not have a Lie group structure compatible with that topology. It should be noted that if $M$ is a smooth CR manifold which is finitely nondegenerate and of finite type at a point $p$, then $\operatorname{Aut}_{\mathrm{CR}}(M, p)$ satisfies a local complete system; this is proved in [E01, KZ02].

We would like to conclude with the following open question. Let $M$ be a connected real-analytic CR manifold such that for every $p \in M$, $\operatorname{Aut}_{\mathrm{CR}}^{\omega}(M, p)$ has a Lie group structure compatible with its topology. Does this imply that $\operatorname{Aut}_{\mathrm{CR}}^{\omega}(M)$ has also a Lie group structure (compatible with its own topology)? Note that the converse implication does not hold. Indeed, for the CR manifold $M$ given in Example 6.4, Aut $_{\mathrm{CR}}^{\omega}(M)=\{\mathrm{id}\}$ whereas, for every $p \in M$, Aut $\mathrm{CR}_{\mathrm{R}}^{\omega}(M, p)$ is infinite-dimensional since $M$ is locally a product of the quadric $Q$ and $\mathbb{R}$.

\section{REFERENCES}

[AF79] Andreotti, A.; Fredricks, G.A. - Embeddability of real analytic Cauchy-Riemann manifolds. Ann. Scuola Norm. Sup. Pisa Cl. Sci. (4) 6 (1979), 285-304.

[BER97] Baouendi, M.S.; Ebenfelt, P.; Rothschild, L.P. - Parametrization of local biholomorphisms of real analytic hypersurfaces. Asian J. Math. 1 (1997), 1-16.

[BER99a] Baouendi, M.S.; Ebenfelt, P.; Rothschild, L.P. - Rational dependence of smooth and analytic CR mappings on their jets. Math. Ann. 315 (1999), 205-249.

[BER99b] Baouendi, M.S.; Ebenfelt, P.; Rothschild, L.P. - Real submanifolds in complex space and their mappings. Princeton Mathematical Series, 47. Princeton University Press, Princeton, NJ, 1999.

[BER00] Baouendi, M.S.; Ebenfelt, P.; Rothschild, L.P. - Convergence and finite determination of formal CR mappings. J. Amer. Math. Soc. 13 (2000), 697-723.

[BJT85] Baouendi, M. S.; Jacobowitz, H.; Treves, F. - On the analyticity of CR mappings. Ann. of Math. (2) 122 (1985), 365-400.

[BMR02] Baouendi, M.S.; Mir, N.; Rothschild, L.P. - Reflection ideals and mappings between generic submanifolds in complex space. J. Geom. Anal. 12 (2002), no. 4, 543-580.

[BRZ04] Baouendi, M.S.; Rothschild, L.P.; Zaitsev, D. — Deformation of generic submanifolds in complex space (in preparation). 
[BM46] Bochner, S.; Montgomery, D. - Locally compact groups of differentiable transformations. Ann. of Math. (2) 47 (1946), 639-653.

[Bo91] Boggess, A. - CR manifolds and the tangential Cauchy-Riemann complex. Studies in Advanced Mathematics. CRC Press, Boca Raton, FL, 1991.

[BS77] Burns, D., Jr.; Shnider, S. - Real hypersurfaces in complex manifolds. Several complex variables. Proc. Sympos. Pure Math., Vol. XXX, Part 2, Williams Coll., Williamstown, Mass., 1975, 141-168. Amer. Math. Soc., Providence, R.I., 1977.

[C32] Cartan, E. - Sur la géométrie pseudo-conforme des hypersurfaces de deux variables complexes I,II. Oevres II, 2 (1932), 1217-1238.

[CM74] Chern, S.S; Moser, J.K. - Real hypersurfaces in complex manifolds. Acta Math. 133 (1974), 219271.

[DF91] Dummit, D.; Foote, R. - Abstract algebra. Prentice Hall, Inc., Englewood Cliffs, NJ, 1991.

[E01] Ebenfelt, P. - Finite jet determination of holomorphic mappings at the boundary. Asian J. Math. 5 (2001), 637-662.

[ELZ03] Ebenfelt, P.; Lamel, B.; Zaitsev, D. - Finite jet determination of local analytic CR automorphisms and their parametrization by 2-jets in the finite type case. Geom. Funct. Anal. 13 (2003), no. 3, 546-573.

[GG73] Golubitsky, M.; Guillemin, V. - Stable mappings and their singularities. Graduate Texts in Mathematics, Vol. 14. Springer-Verlag, New York-Heidelberg, 1973.

[H97] Han, C.-K. - Complete differential system for the mappings of CR manifolds of nondegenerate Levi forms. Math. Ann. 309 (1997), 401-409.

[KZ02] Kim, S.-Y.; Zaitsev, D. - Equivalence and embedding problems for CR-structures of any codimension. Preprint (2002).

[KZ03] Kim, S.-Y.; Zaitsev, D. - Remarks on the rigidity of CR-manifolds (in preparation).

[Ko72] Kobayashi, S. - Transformation groups in differential geometry. Ergebnisse der Mathematik und ihrer Grenzgebiete, Band 70. Springer-Verlag, New York-Heidelberg, 1972.

[Kw01] Kowalski, R.T. - Rational jet dependence of formal equivalences between real-analytic hypersurfaces in $\mathbb{C}^{2}$. Preprint (2001); http://arXiv.org/abs/math.CV/0108165.

[T67] Tanaka, N. - On generalized graded Lie algebras and geometric structures I. J. Math. Soc. Japan 19 (1967), 215-254.

[Tu88] Tumanov, A.E. - Extension of CR-functions into a wedge from a manifold of finite type. (Russian). Mat. Sb. (N.S.) 136 (178) (1988), 128-139; translation in Math. USSR-Sb. 64 (1989), 129-140.

[V74] Varadarajan, V. S. - Lie groups, Lie algebras, and their representations. Prentice-Hall Series in Modern Analysis. Prentice-Hall, Inc., Englewood Cliffs, N.J., 1974.

[Z95] Zaitsev, D. - On the automorphism groups of algebraic bounded domains. Math. Ann. 302 (1995), 105-129.

[Z97] Zaitsev, D. - Germs of local automorphisms of real analytic CR structures and analytic dependence on the $k$-jets. Math. Res. Lett. 4 (1997), 823-842.

M.S. Baouendi and L. P. Rothschild: Department of Mathematics, 0112, Univ. of California, San Diego, La Jolla, CA 92093-0112, USA

E-mail address: sbaouendi@ucsd.edu, Irothschild@ucsd.edu

J. Winkelmann: Institut Élie Cartan (Mathématiques), Université Henri Poincaré Nancy 1, B.P. 239, F-54506 Vandeuvre-LÈs-Nancy Cedex, France

E-mail address: jwinkel@member.ams.org

D. Zaitsev: School of Mathematics, Trinity College, Dublin 2, Ireland

E-mail address: zaitsev@maths.tcd.ie 